\title{
COMPORTAMENTO SÓCIO-SEXUAL E DE MANUTENÇÃO DE TOUROS NELORE DURANTE A ESTAÇÃO DE MONTA
}

\author{
SOCIAL HIERARCHY IN NELLORE BULLS AND SEXUAL AND MAINTENANCE \\ ACTIVITIES DURING BREEDING SEASON
}

\author{
Costa-e-Silva, E.V. ${ }^{1 *}$, Sereno, J.R.B. ${ }^{2}$, Vasconcelos, J.T. ${ }^{3}$, Zúccari, C.E.S.N. ${ }^{1}$ \\ e Paranhos-da-Costa, M.J.R. ${ }^{4}$
}

\author{
${ }^{1}$ Laboratório de Reprodução Animal. FAMEZ. Universidade Federal de Mato Grosso do Sul. CP 549. CEP \\ 79070-900. Campo Grande, MS. Brasil. *licsilva@nin.ufms.br \\ ${ }^{2}$ Embrapa Cerrados. Planaltina, DF. 73310-970. Brasil. \\ ${ }^{3}$ FAMEZ/UFMS. PIBIC/CNPq. Brasil. \\ ${ }^{4}$ Departamento de Zootecnia. FCAV-UNESP. Jaboticabal, SP. Brasil.
}

\section{PALAVRAS ChaVE ADICIONAIS}

Bos indicus. Dominância social. Pastejo. Ruminação. Zebu.

\section{RESUMO}

As proposições de manejo de touros zebuínos têm sido feitas sem avaliação do impacto sobre o seu comportamento e bem-estar. O objetivo deste estudo foi avaliar se o aumento na atividade sexual de touros Nelore altera o tempo despendido em atividades de manutenção e se isto depende do status social do touro. Seis touros foram distribuídos em três lotes: 1) um touro e 60 vacas, 2) dois touros e 120 vacas em cio natural e 3) três touros e 180 fêmeas, induzindo o cio em 50 delas. Foi adotada amostragem focal com observação contínua por 120 horas, registrando-se o tempo dedicado às atividades sexuais e de manutenção. A posição hierárquica influenciou o tempo despendido em atividades sexuais, que foi maior para touros sob alta pressão de cio que os de baixa. Como esperado observou-se que o status social dos touros interferiu na definição do tempo despendido em atividades sexuais, com detrimento das atividades de manutenção.

\section{SUMMARY}

Changes in the bull:cow proportion in natural breeding have been proposed, but there are not evaluations about the effect of these changes in the behaviour of Nellore bulls. The objective of this study was to test the hypothesis that the increase of sexual activity of Nellore bulls, due to the highest

Recibido: 25-1-08. Aceptado: 22-10-08.

\section{AdDitional KeYWORDS}

Bos indicus. Social dominance. Grazing. Rumination. Zebu bulls.

heat incidence $(\mathrm{HHI})$, changes the time of general activity of those animals depending on their social status in the group. Six Nellore bulls were distributed in three groups: one bull in a single-sire breeding group (1bull:60cows) and two in multiplesire breeding groups - one with 2 bulls and 120 cows (cycling naturally) and the other group with 3 bulls and 180 females, 50 of which were synchronised. The behaviour of these animals was recorded during 120 hours, using continuous and direct observation with animal focal sampling. The dominance order influenced the time spent with sexual activities. Bulls under high heat incidence presented the largest sexual activities and the smaller grazing, agonistic interactions and other activities, there was no difference in rumination.

\section{INTRODUÇÃO}

O manejo reprodutivo do rebanho de corte brasileiro caracteriza-se por sistemas extensivos, com estações de monta definidas nos meses de primavera ou verão, sendo que cerca de $95 \%$ do processo reprodutivo ainda ocorre por monta natural (Costa e Silva, 1995). Em decorrência disto, a demanda anual por touros é enorme, considerando 
a proporção touro:vaca (PTV) 1:25 (média no Brasil), aproximadamente 300 mil animais (Rosa, 2007). Uma das propostas de manejo que vem sendo feita é a redução da PTV, no sentido de minimizar os custos da reposição anual de touros. Estima-se que a redução da PTV de 1:25 para 1:40 diminua em 15\% os custos do bezerro ou 14,6 dólares por bezerro no sistema de produção brasileiro (Fonseca et al., 1991). No entanto, é possível alcançar maiores estimativas, quanto maior for a redução de touros. Por exemplo, em regiões como o Pantanal brasileiro onde se utiliza PTV de 1:10, alterá-la para 1:25 ou 1:40 proporciona uma queda da ordem de $60 \%$ e $75 \%$ nos custos de produção do bezerro, respectivamente (Sereno et al., 2000). A garantia de uma relação custo beneficio positiva é essencial que não haja perdas na eficiência reprodutiva. Diversos autores brasileiros têm testado menores PTV com sucesso. Inicialmente, Crudeli et al. (1990) e Costa e Silva (1994) avaliaram PTV de 1:40 e 1:60 em sistemas de acasalamento individual, em touros Nelore, e registraram média de $90 \%$ de prenhez aos 120 e 90 dias de estação de monta, respectivamente. Chegando à PTV de 1:80 (Costa e Silva, 1994; Pineda et al., 2000) e até 1:100 (Santos et al. , 2004). Pineda et al. (2000) e Santos et al. (2004) registraram a perda de peso de 5 e $9,1 \%$, respectivamente, mas não correlacionaram esta perda com a PTV adotada.

Estudos sobre o efeito de diferentes pressões de cio sobre o comportamento sócio-sexual, bem como nas atividades gerais de manutenção, são ainda escassos. Touros europeus adultos, de raças de corte, mantidos numa PTV média de 1:28,6 não apresentaram variação significativa no padrão diurno de pastejo durante a estação de monta (Raadsma et al., 1983/1984 e Boyd et al., 1989). No entanto, em se tratando de PTV tão baixas, quanto as que têm sido propostas para o Nelore no Brasil (Santos et al., 2004; Pineda et al., 2000), surge a preocupação com o impacto negativo sobre as atividades gerais e as possíveis conse- qüências sobre a integridade reprodutiva dos touros.

O objetivo deste trabalho foi testar a hipótese de que o aumento na atividade sexual de touros Nelore, decorrente do aumento da pressão de cio, altera o tempo despendido nas atividades de manutenção desses animais, avaliando a predição de que tal alteração depende do status social dos touros.

\section{MATERIAL E MÉTODOS}

Este estudo foi realizado numa propriedade situada na transição entre o Pantanal e o Planalto Central no Estado do Mato Grosso do Sul (latitude 1951'S, longitude 56⒌'W e altitude $125 \mathrm{~m}$ ). A região apresenta médias anuais de pluviosidade de 1182 mm concentrada no verão, temperatura de $25^{\circ} \mathrm{C}$ (com variações entre 19,7 e $32^{\circ} \mathrm{C}$ ) e umidade relativa do ar de $82 \%$ (com variação entre 48 a 96\%, no inverno e verão, respectivamente).

Após exame andrológico e testes de libido, de um total de 93 machos Nelore registrados, foram selecionados 6 touros adultos, com experiência sexual prévia, com média de idade de 7 anos, média de perímetro escrotal de $38,25 \mathrm{~cm}$. No teste de libido, baseando-se na metodologia proposta por Chenoweth (1981), foram classificados como de libido média, com moda seis na pontuação. Organizou-se três lotes: a) um em acasalamento simples (um touro e 60 vacas), b) dois em acasalamento múltiplo, sendo um com dois touros e 120 fêmeas em cio natural (baixa incidência de cio) e c) três touros e 180 fêmeas, das quais 50 foram submetidas à sincronização de cio (alta incidência de cio). As vacas Nelore foram distribuídas proporcionalmente nos lotes considerando a categoria: primíparas e pluríparas, após exame ginecológico prévio, por meio de palpação retal, evidenciandose atividade cíclica dos ovários. Todas estavam em boa condição corporal (moda para escore 4, escala de 1 a 5), período

Archivos de zootecnia vol. 59, núm. 227, p. 322. 
puerperal mínimo de 60 dias e peso médio de $372,78 \mathrm{~kg}$, no início da estação. No lote de alta incidência de cio, a sincronização do ciclo foi realizada pela aplicação de $3 \mathrm{mg}$ de Norgestomet e $5 \mathrm{mg}$ de valerato de estradiol, via IM, logo após a colocação do implante auricular contendo $6 \mathrm{mg}$ de Norgestomet (Syncro-mate $ß 囚$ ). As fêmeas sincronizadas foram introduzidas no lote logo após o implante auricular. As observações iniciaramse um dia depois.

Os lotes foram formados 30 dias antes do início das observações e mantidos em pastos (Brachiaria brizantha) com pouca declividade, com boa distribuição de sombra. A média de taxa de lotação utilizada foi de 1,17 U.A./ha. A estação de reprodução foi iniciada em novembro, época adequada às características da região e teve duração de 120 dias. As observações foram realizadas por duas estações reprodutivas consecutivas, no verão (dezembro/janeiro).

A metodologia de observação adotada foi a de coleta contínua com amostragem focal por cinco dias consecutivos, 24 horas por dia, sendo um observador para cada touro. Os observadores trabalharam em turnos de seis horas/dia, montados a cavalo, munidos de binóculos, lanterna e equipamento adequado para garantir os registros, mesmo sob chuva. Para facilitar a visão noturna, as observações foram realizadas próximas das noites de lua cheia (até cinco dias antes e cinco depois).

Registrou-se o tempo despendido em: atividades sexuais (SEX), interações agonísticas (INT), pastejo (PAST), ruminação (RUM) e outras atividades (OUT - composta, principalmente, por ócio e deslocamento).

A posição social de cada macho por lote de acasalamento múltiplo foi definida por meio das observações dos confrontos (ataques e fugas) entre eles, definindo-os como: dominante (Do) e subordinados (Sb). O touro do lote de acasalamento individual foi classificado como sem hierarquia (SH).

O banco de dados foi organizado discriminando-se o tempo de cada atividade em períodos de 3 horas ao longo do dia (definidos a partir da hora zero em: 0-3 h, 3-6 h, 69 h ..., 21-24 h). A análise de variância, pelo Procedimento GLM do pacote estatístico SAS (2005), considerou efeitos fixos de pressão de cio (I), ordem na hierarquia (H), ordem do dia de observação (D), período do dia (T), ocorrência de serviço completo $(\mathrm{O})$ e as interações de interesse, como apresentado no modelo a seguir:

$\mathrm{Y}_{\mathrm{ijklm}}=\mu_{\mathrm{ijklm}}+\mathrm{H}_{\mathrm{i}}+\mathrm{I}_{\mathrm{i}}+\mathrm{D}_{\mathrm{k}}+\mathrm{T}_{1}+\mathrm{O}_{\mathrm{m}}+\mathrm{HI}_{\mathrm{ij}}+\mathrm{HD}_{\mathrm{ik}}+\mathrm{HT}_{\mathrm{jl}}$ $+I D_{j k}+I T_{i l}+D T_{k l}+D O_{k m}+T_{I m}+\varepsilon_{i j k l m}$

Emque:

$Y_{\mathrm{ijk} / \mathrm{m}}=$ tempo gasto pelos touros em cada uma das atividades (SEX, PAST, RUM, INT, OUT);

$\mu_{i j k \mathrm{k}}=$ média geral;

$\mathrm{H}_{\mathrm{i}}=$ efeito fixo da i-ésima ordem na hierarquia (i= $0,1,2)$;

$\mathrm{I}_{\mathrm{j}}=$ efeito fixo da j-ésima incidência de cio $(\mathrm{j}=1,2)$;

$D_{k}=$ efeito fixo da k-ésima ordem do dia de observação $(k=1,2,3,4,5)$;

$T_{1}=$ efeito fixo do l-ésimo período do dia $(I=1,2,3$, $4,5,6,7,8)$;

$\mathrm{O}_{\mathrm{m}}=$ efeito fixo da m-ésima ocorrência de serviço completo no lote $(m=0,1)$;

$\mathrm{HI}_{\mathrm{ij}}=$ efeito da interação entre a i-ésima ordem na hierarquia com a j-ésima incidência de cio;

$H D_{i k}=$ efeito da interação entre a i-ésima ordem na hierarquia com a k-ésimo ordem do dia de observação;

$H T_{\mathrm{jl}}=$ efeito da interação entre a i-ésima ordem na hierarquia e o l-ésimo período do dia;

$I D_{j k}=$ efeito da interação entre a j-ésima incidência de cio com a k-ésima ordem do dia de observação;

$\mathrm{IT}_{\mathrm{il}}=$ efeito da interação entre a j-ésima incidência de cio e o l-ésimo período do dia;

$\mathrm{DT}_{\mathrm{kl}}=$ efeito da interação entre a k-ésima ordem do dia de observação e o l-ésimo período do dia;

$\mathrm{DO}_{\mathrm{km}}=$ efeito da interação entre a k-ésima ordem do dia de observação e da m-ésima ocorrência de serviço completo no lote;

$\mathrm{TO}_{\mathrm{Im}}=$ efeito da interação entre o l-ésimo período do dia e da m-ésima ocorrência de serviço completo no lote;

$\varepsilon_{i \mathrm{ijkm}}=$ erro aleatório.

Para algumas variáveis foram calculados os contrastes de médias para compa-

Archivos de zootecnia vol. 59, núm. 227, p. 323. 
ração dos efeitos de:

- Ordem de hierarquia: (Do) vs. (Sb); Do $v s$. Sb sob alta incidência de cio; Do vs. Sb sob baixa incidência de cio.

-Efeito da competição: (SH) vs. (Do); (SH) vs. (Sb);

- Períodos do dia: noite vs. dia (T1+T2+T7+T8) vs. (T3+T4+T5+T6); noite (6-24 horas) vs. madrugada (0-6 horas) (T1+T2) vs. (T7+T8); manhã vs. tarde (T3+T4) vs. (T5+T6).

As comparações de médias entre ordem do dia foram realizadas pelo teste de Duncan e entre as médias de incidência de cio e ocorrência ou não de serviço completo, pelo teste $t$ de Student (Sampaio, 1998).

\section{RESULTADOSEDISCUSSÃO}

O tempo médio de observação por touro foi de 113 horas e 42 minutos, totalizando 682 horas e 16 minutos. O tempo médio diário despendido em atividades sexuais (SEX), pastejo (PAST), ruminação (RUM), interações agonísticas (INT) e outras atividades (OUT), expressos em minutos pordia(24horas)foi: 353,83 $\pm 48,71$ (25,05\%), $274,67 \pm 25,88$ (19,60\%), 276,50 $\pm 37,41$

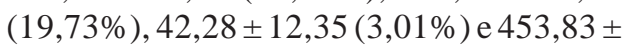
48,73(32,39\%), respectivamente.

O modelo de análise foi significativo

para as seguintes variáveis: SEX (Anova: $\left.\mathrm{p}=0,0001 ; \mathrm{R}^{2}=0,68 ; \mathrm{CV}=43,19\right), \mathrm{PAST}$ (Anova: $\mathrm{p}=0,0001 ; \mathrm{R}^{2}=0,63 ; \mathrm{CV}=45,49$ ), INT (Anova: $p=0,0015 ; R^{2}=0,55 ; C V=79,81$ ) e OUT (Anova: $\mathrm{p}=0,0412 ; \mathrm{R}^{2}=0,49 ; \mathrm{CV}=$ 51,36), mas não para RUM(Anova: $p=0,0913$, $\left.\mathrm{R}^{2}=0,47 ; \mathrm{CV}=67,96\right)$.

A ordem hierárquica afetou significativamente $(p=0,0290)$ o tempo despendido com atividades sexuais (tabela I). Os contrastes entre as médias de touros em função das classes hierárquicas (dominantes, subordinados e sem hierarquia) mostraram efeito significativo apenas entre as médias de SEX dos dominantes vs subordinados $(p=0,0049)$. Touros sob alta pressão de cio utilizaram maior tempo em atividades sexual $(p=0,0009)$ e menor em pastejo $(p=0,0152)$, interações agonísticas $(p=0,0002)$ e outras atividades ( $\mathrm{p}=0,0513)$, não interferindo no tempo de ruminação $(\mathrm{p}>0,05)$ (tabela II). A ordem do dia de observação influenciou ( $\mathrm{p}=$ 0,0015 ) apenas o tempo despendido com atividades sexuais, com diminuição gradativa nesta atividade do primeiro ao quinto dia de observação (figura 1).

Os períodos do dia influenciaram significativamente $(p=0,0046)$ apenas o tempo despendido com pastejo. Ao testar os efeitos de períodos do dia observou-se, pelos contrastes (figura 2), maior atividade

Tabela I. Tempo médio (minutos por período de 3 horas) despendido por touros Nelore com atividades sexuais (SEX), pastejo (PAST), ruminação (RUM), interações agonísticas (INT) e outras atividades (OUT) e respectivos erros padrão e porcentagens em relação ao tempo total (turno de 180 minutos), de acordo com a ordem hierárquica. (Average time (minutes/three hours periods) spent by Nellore bulls with sexual activities (SEX), grazing behaviour (PAST), rumination (RUM), agonistic interaction (INT) and resting time (OUT) and respective standard error and percent accord to period of 180 minutes, by social hierarchy).

\begin{tabular}{|c|c|c|c|c|c|c|c|c|c|c|}
\hline & SEX & $\%$ & PAST & $\%$ & RUM & $\%$ & INT & $\%$ & OUT & $\%$ \\
\hline D & $51,89 \pm 5,45$ & 29,72 & $31,37 \pm 3,45$ & 17,97 & $31,84 \pm 4,09$ & 18,24 & $4,82 \pm 1,28$ & 2,44 & $54,69 \pm 5,58$ & 31,32 \\
\hline $\mathrm{S}$ & $39,68 \pm 3,94$ & 22,57 & $35,07 \pm 3,39$ & 19,95 & $35,04 \pm 3,86$ & 19,93 & $3,93 \pm 0,84$ & 2,24 & $62,06 \pm 4,92$ & 35,31 \\
\hline SH & $34,62 \pm 6,55$ & 19,45 & $44,90 \pm 6,13$ & 25,22 & $48,95 \pm 8,97$ & 27,50 & $9,00 \pm 4,61$ & 5,06 & $40,57 \pm 7,42$ & 22,78 \\
\hline MG & $42,88 \pm 2,92$ & 24,35 & $35,59 \pm 2,30$ & 20,21 & $36,46 \pm 2,84$ & 20,70 & $5,46 \pm 1,01$ & 2,92 & $55,72 \pm 3,34$ & 31,63 \\
\hline
\end{tabular}

Ordem de dominância: D: Dominantes; S: Subordinados; Sh: sem hierarquia; MG: Media geral.

Archivos de zootecnia vol. 59, núm. 227, p. 324. 
Tabela II. Tempo médio (minutos/por turno de 3 horas) despendido por touros Nelore com comportamento sexual e de manutenção e respectivos erros padrão e porcentagens em função das classes de incidência de cio. (Average time (minutes/3 hours periods) spent by Nellore bulls with sexual and maintenance behaviour and respective standard error and percent by heat incidence classes).

\begin{tabular}{|c|c|c|c|c|c|c|c|}
\hline & $\begin{array}{r}\text { Atividade } \\
\text { sexuais }\end{array}$ & $\%$ & Pastejo & $\%$ & $\begin{array}{l}\text { Ruminação } \\
\text { agonísticas\% }\end{array}$ & $\begin{array}{l}\text { Interações } \\
\text { atividades \% }\end{array}$ & Outras \\
\hline A & $59,17 \pm 4,86$ & 33,27 & $26,04 \pm 2,80$ & 14,64 & $28,37 \pm 3,50 \quad 15,95$ & $1,74 \pm 0,42 \quad 0,98$ & $62,53 \pm 4,9435,16$ \\
\hline B & $29,45 \pm 3,00$ & 16,92 & $43,45 \pm 3,35$ & 24,96 & $43,13 \pm 4,22 \quad 24,78$ & $7,94 \pm 1,79 \quad 4,56$ & $50,10 \pm 4,48 \quad 28,78$ \\
\hline MG & $\quad 42,88 \pm 2,9$ & & $35,59 \pm 2,3$ & & $36,46 \pm 2,84$ & $5,46 \pm 1,01$ & $55,72 \pm 3,34$ \\
\hline
\end{tabular}

A: Incidência de cio alta; B: Incidência de cio baixa; MG: Média geral.

de pastejo durante o dia $(\mathrm{p}=0,0219)$ e principalmente à tarde $(\mathrm{p}=0,0002)$ e que o pastejo ocorreu por mais tempo a noite do que na madrugada. Apesar da não significância do período do dia sobre o tempo despendido com interações agonísticas, detectou-se, quando as frações de 180 minutos foram agrupadas, diferença significativa na comparação noite $\mathrm{x}$ dia $(\mathrm{p}=0,0079)$, com maior concentração diurna de interações agonísticas (figura 3). Da mesma forma para outras atividades, que ocorreu por maior tempo na madrugada (0-6 h) quando comparada com a noite $(\mathrm{p}=0,0010)$, provavelmente

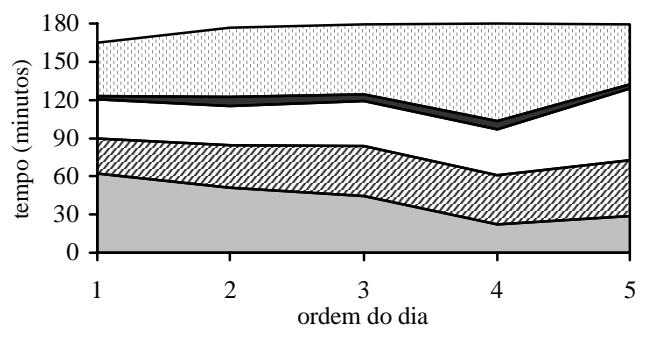

devido ao deslocamento dos animais após a meia-noite para as áreas de descanso (malhadouros), onde permaneciam até o amanhecer. Houve concentração de ócio no período entre 3 e 6 horas da manhã $(p=$ 0,0306). Também para as atividades sexuais, observou-se variação significativa $(\mathrm{p}=$ 0,0335 ) entre as médias obtidas para noite (6-24 h) e madrugada (0-6 h). A distribuição das atividades dos touros no decorrer do dia é apresentada na figura 3.

A ocorrência de serviço completo afetou significativamente o tempo gasto com atividades sexuais $(\mathrm{p}=0,0027)$, mas não

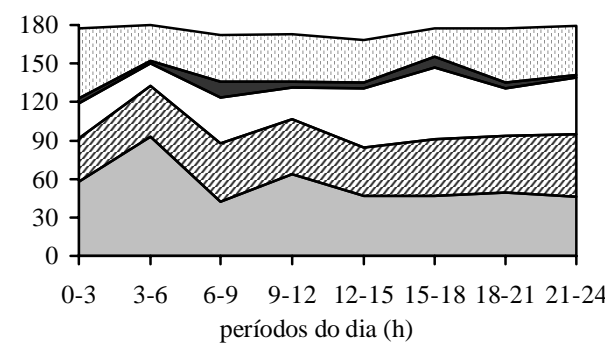

atividades sexuais 四 pastejo interações agonísticas $\quad$ Outras atividades

$\square$ ruminação
Figura 1. Distribuição do comportamento sexual e de manutenção realizados por touros Nelore em estação de monta no decorrer de 24 horas de acordo com a ordem do dia de observação. (Distribution of sexual and maintenance behaviour by Nellore bulls during the breeding season during 24 hours by observation day order).
Figura 2. Distribuição do comportamento sexual e de manutenção realizados por touros Nelore em estação de monta no decorrer de 24 horas durante a estação reprodutiva. (Distribution of sexual and maintenance behaviour of Nellore bulls through 24 hours of observation during breeding season). 


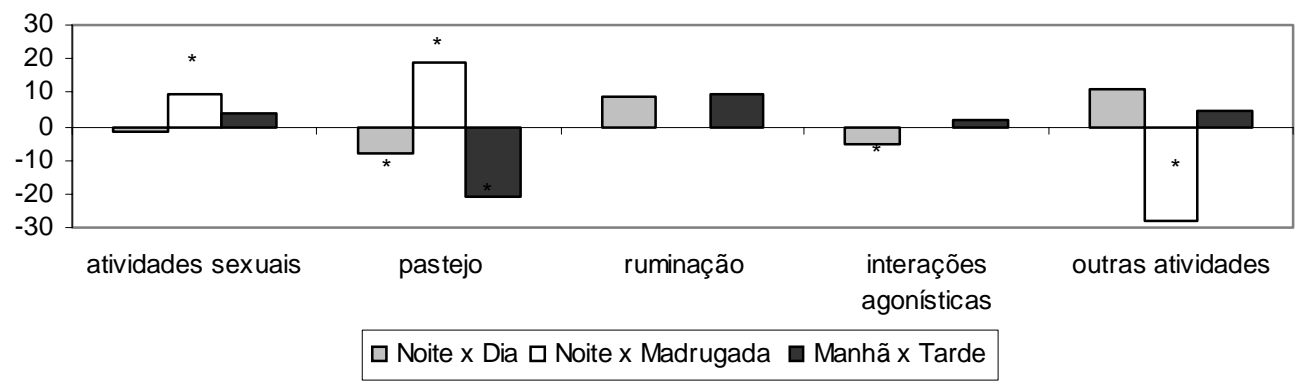

Figura 3. Contrastes de médias entre o tempo de atividades sexuais e de manutenção de touros Nelore, de acordo com os períodos do dia: noite (18-6 h) vs. dia (6-18 h); manhã (6$12 \mathrm{~h})$ vs. tarde $(12-18 \mathrm{~h})$; noite $(18-24 \mathrm{~h})$ vs. madrugada $(0-6 \mathrm{~h}) .{ }^{*} p<0,05$. (Mean contrasts among time of sexual and maintenance behaviour of Nellore bulls, according with the periods of day: (night ( 6 p.m. to 6 a.m.) vs. day ( 6 a.m. to 6 p.m.); morning ( 6 a.m. to 12 p.m.) vs. afternoon (12 p.m. to 6 p.m.); night (6 p.m. to 12 a.m.) vs. morning (12 a.m. to 6 a.m.)). *p<0.05.

influenciou pastejo, ruminação, interações agonísticas e outras atividades ( $\mathrm{p}>0,05)$. As médias de tempo gasto nas diferentes atividades observadas estão discriminadas na tabela III.

Não foi observada interação significativa entre hierarquia e incidência de cio $\left(\mathrm{H}^{*} \mathrm{I}\right)$ para o tempo gasto nas diferentes atividades observadas. Observou-se interação significativa entre hierarquia e ordem do dia para interações agonísticas ( $p=0,0488)$, mas não para atividades sexuais, pastejo, ruminação e outras atividades $(p>0,05)$, figura 4 . A interação entre hierarquia e período do dia foi significativa para pastejo $(p=0,0359)$ e interações agonísticas ( $\mathrm{p}=0,0006)$, mas não para atividades sexuais, ruminação e outras atividades ( $p>0,05)$, figura 5 . Observou-se interação significativa entre incidência de cio e ordem do dia de observação para as atividades sexuais $(\mathrm{p}=0,0090)$ e pastejo $(\mathrm{p}=$ $0,0001)$. Estes resultados são ilustrados na figura 6A. Ruminação, interações agonísticas e outras atividades não apresentaram variação significativa ( $p>0,05)$ em decorrência dessa interação. Observou-se interação significativa entre incidência de cio e períodos do dia para atividades sexuais ( $p=0,0152)$, pastejo $(p=0,0504)$ e interações agonísticas ( $p=0,0493)$ (figura 6B). Não foi observada interação significativa entre ordem do dia de observação e período do

Tabela III. Tempo médio (minutos/turno de 3 horas) despendido por touros Nelore em comportamento sexual e de manutenção e respectivas porcentagens em relação ao tempo total (turno de 180 minutos) de acordo com a ocorrência de serviço completo (SC) no lote. (Average time (minutes/3 hours periods) spent by Nellore bulls with sexual and maintenance behaviour and respective standard error and percent by complete service occurrence).

\begin{tabular}{|c|c|c|c|c|c|c|c|}
\hline & $\begin{array}{c}\text { Atividades } \\
\text { sexuais }\end{array}$ & S $\%$ & Pastejo & $\%$ & $\begin{array}{l}\text { Ruminação } \\
\text { agonísticas \% }\end{array}$ & $\begin{array}{l}\text { Interações } \\
\text { atividades \% }\end{array}$ & Outras \\
\hline $\mathrm{N}$ & $39,43 \pm 2,59^{b}$ & 17,38 & $39,40 \pm 2,71^{a}$ & 22,50 & $39,62 \pm 3,41^{\mathrm{a}} 22,63$ & $6,02 \pm 1,31^{\mathrm{a}} 3,44$ & $59,64 \pm 4,07^{a} 34,06$ \\
\hline O & $81,40 \pm 6,45^{a}$ & 45,75 & $23,81 \pm 3,93^{b}$ & 13,38 & $26,70 \pm 4,64^{\mathrm{a}} 15,01$ & $2,43 \pm 0,84^{\mathrm{b}} 1,37$ & $43,57 \pm 5,04^{\text {a }} 24,49$ \\
\hline
\end{tabular}

$\mathrm{N}$ : Não ocorrência de SC no lote; O: Ocorrência de SC no lote.

*letras diferentes nas colunas indicam diferença significativa em nível de 5\% pelo teste $t$ de Student.

Archivos de zootecnia vol. 59, núm. 227, p. 326. 

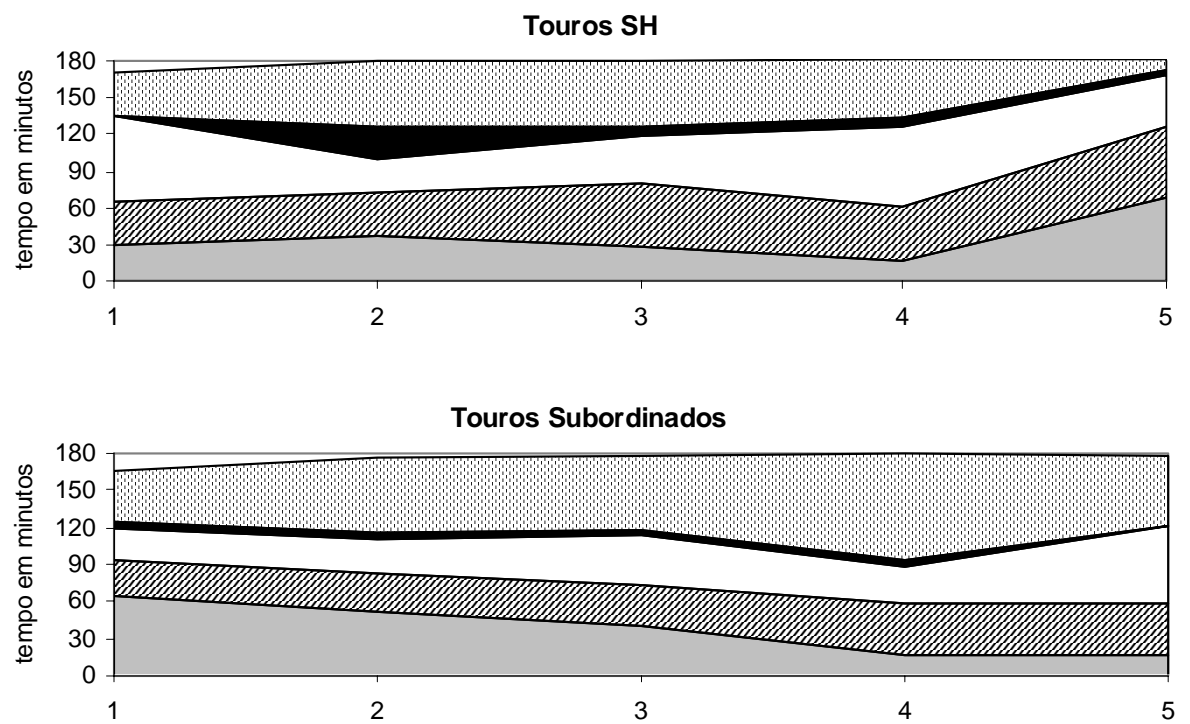

Touros Dominantes

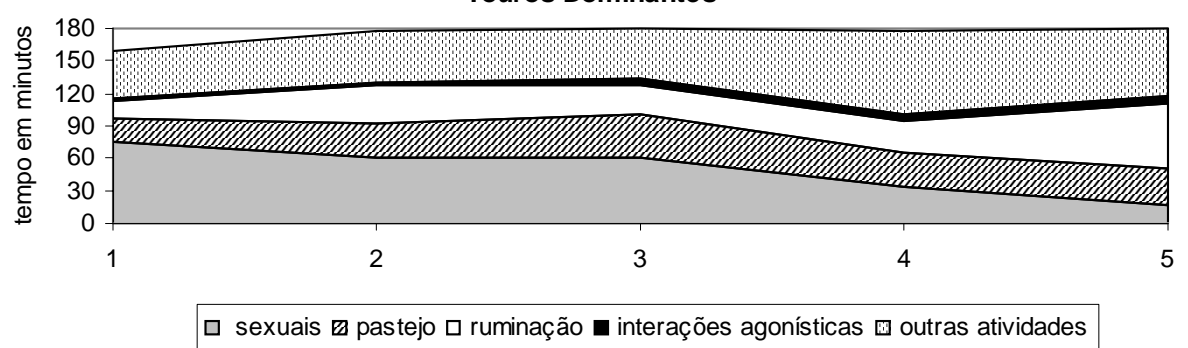

Figura 4. Distribuição das atividades observadas em touros Nelore durante a estação de monta, de acordo com ordem de dominância e ordem do dia de observação. (Activities observed in Nellore bulls during the breeding season by social hierarchy and observation day.

dia ou com ocorrência de serviço completo para nenhuma das variáveis testadas. A interação período do dia e ocorrência de serviço completo também não foi significativa para as variáveis testadas.

$\mathrm{O}$ aumento no tempo despendido em atividades sexuais pode ter reflexos negativos no tempo que os touros despenderam nas atividades de manutenção, principalmente com a diminuição dos tempos de pastejo e outras atividades (que englobava ócio e deslocamento). Todavia, é evidente que tais efeitos não foram diretos nem absolutos, sendo dependente de vários fatores, dentre eles: da posição do touro na hierarquia de dominância, da disponibilidade de fêmeas em cio e da própria ocorrência de atividades sexuais. No geral, as atividades sexuais ocuparam em média 25,05\% das 24 horas do dia. Percentual menor foi observado por Raadsma et al. (1983/1984) com touros cruzados europeus na Austrália, registrando 11,9\% do tempo de observação, que foi realizada entre 5 e 19 horas.

Archivos de zootecnia vol. 59, núm. 227, p. 327. 

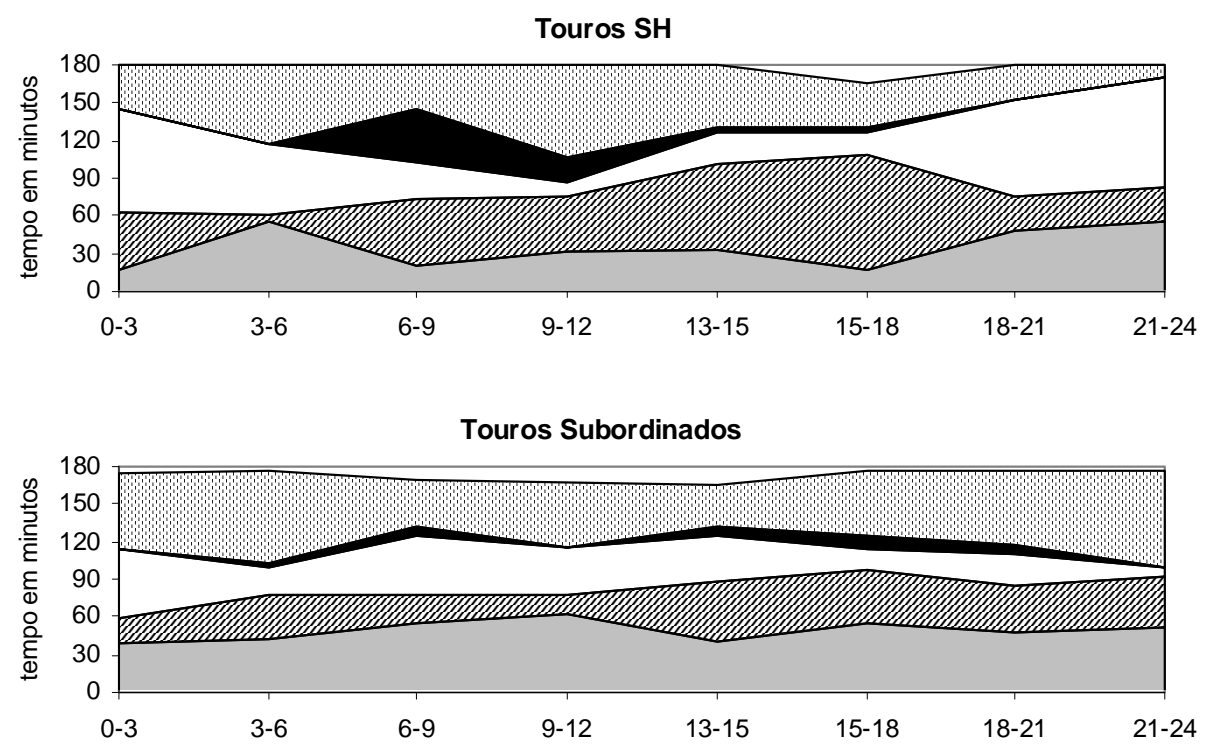

Touros Dominantes

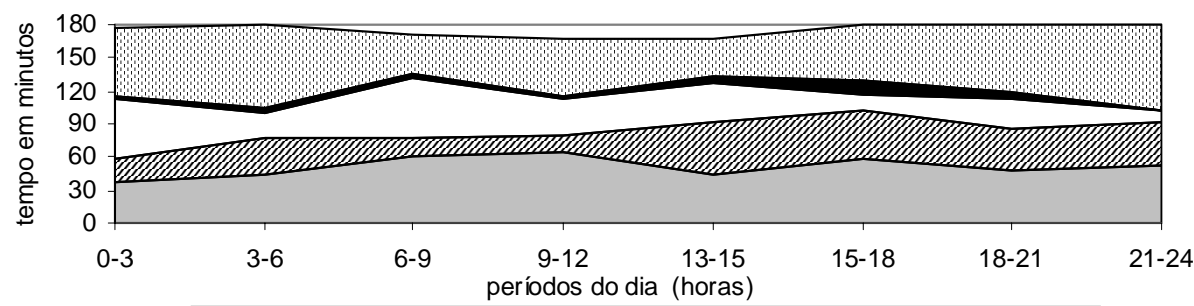

$\square$ sexuais $\square$ pastejo $\square$ ruminação $\square$ interações agonísticas . outras atividades

Figura 5. Distribuição das atividades observadas em touros Nelore durante a estação de monta, de acordo com ordem de dominância e período do dia. (Activities observed in Nellore bulls during the breeding season according to social hierarchy and period of the day).

Os touros tiveram em média 0,83 e 8,6 fêmeas em cio por dia nos lotes de baixa e alta incidência de cio, respectivamente. Como esperado, a maior incidência de cio teve efeito direto no tempo despendido em atividades sexuais, aumentando-o. Nesta condição, os touros ocuparam quase o dobro do tempo daquele ocupado sob baixa pressão de cio. Valor muito inferior foi encontrado no estudo de Raadsma et al. (1983/ 1984), com $2,7 \%$ do tempo em atividade sexual, quando havia em média 0,37 fêmeas em cio por dia. No entanto, mesmo, sob baixa incidência de cio, o percentual de tempo diário demandado em atividades sexuais pelos touros, $16,92 \%$, foi maior. Os resultados de Blockey (1978), com touros Angus, mostraram que quando não havia fêmeas em cio no lote, os touros despendiam $3,3 \%$ do tempo em atividades sexuais, percentual muito baixo. Essas diferenças podem ter ocorrido em decorrência de variações metodológicas no período de observação: cinco dias não consecutivos

Archivos de zootecnia vol. 59, núm. 227, p. 328. 

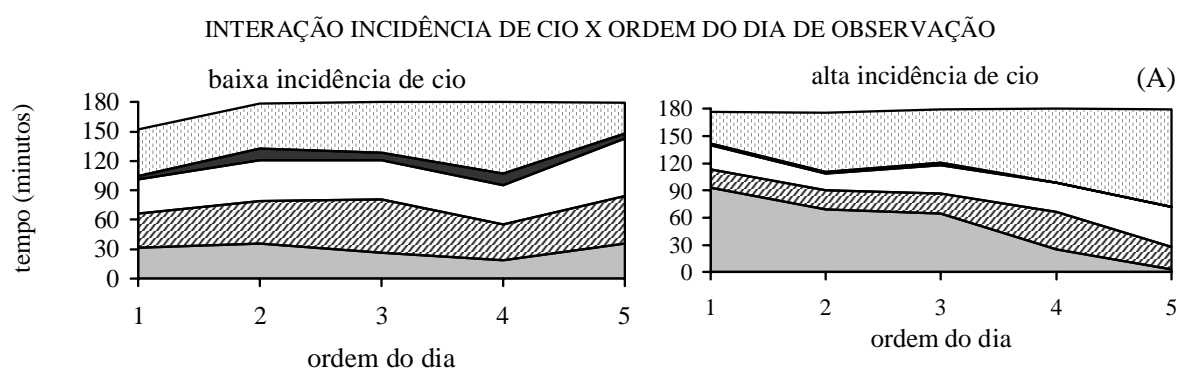

INTERAÇÃO INCIDÊNCIA DE CIO X PERÍODOS DO DIA
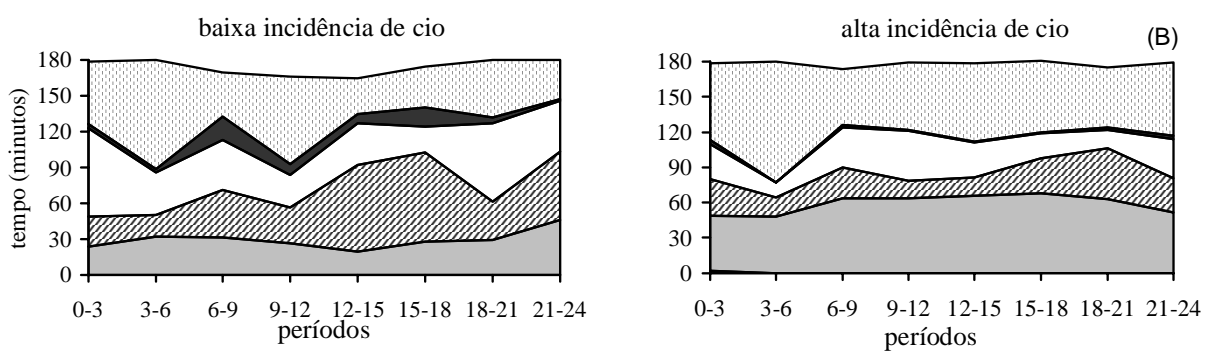

\begin{tabular}{|lll|}
$\square$ atividades sexuais & Q pastejo & $\square$ ruminação \\
$\square$ interações agonísticas & outras atividades & \\
\hline
\end{tabular}

Figura 6. Distribuição do comportamento sexual e de manutenção por touros Nelore em estação de monta: (A) incidência de cio e a ordem do dia de observação; (B) incidência de cio e o período do dia. (Distribution of sexual and maintenance behaviour of Nellore bulls during the breeding season: (A) heat incidence and observation day; (B) heat incidence and period of the day).

de observação e porque os touros ficaram muito tempo sem apresentar serviço completo, 21\% do tempo total de observação. O autor não fez referência ao tempo gasto nessas atividades quando havia fêmeas em cio. Neste estudo sempre houve fêmeas em cio e, sendo os dias de observação consecutivos, provavelmente, mesmo nos períodos em que não houve ocorrência de serviço completo, os touros apresentavam atividades sexuais em relação às vacas em proestro, período no qual já há aumento da estimulação, com 15,55\% do tempo despendido em atividades sexuais. Com a ocorrência de serviço completo no lote, independente de qual dos touros tenha executado a cópula, houve aumento, ainda maior, do tempo despendido em atividades sexuais. Provavelmente isto ocorreu porque as interações entre fêmeas no grupo sexualmente ativo (SAG) sejam estimulantes, tanto para o próprio touro que tenha executado montas como para outros que a assistiram, estimulados pela visão e pelo olfato (Blockey, 1979; Mader e Price, 1984).

A interação significativa entre incidência de cio e dia provavelmente foi observada em decorrência do método de sincronização utilizado no lote de alta incidência de cio, o que levou a uma maior concentração de fêmeas receptivas 48 a 72 horas depois da retirada do implante (Valle et al., 1994), que nesse estudo coincidiu com o primeiro e segundo dias de observação.

O tempo médio diário de pastejo nos touros foi menor do que o registrado na literatura, ocupando apenas 19,60\% do dia. Raadsma et al.(1983/1984) registraram 45\%

Archivos de zootecnia vol. 59, núm. 227, p. 329. 
do tempo total de observação (entre 5 e 19 horas) dedicado ao pastejo, em touros cruzados europeus, enquanto que Boyd et al. (1989) registraram que touros Angus gastaram $36 \%$ do tempo pastando. Os resultados deste estudo foram inferiores aos observados no Brasil para a raça Nelore: Machado Filho et al. (1993a) encontraram 8 horas/dia de tempo médio de pastejo para vacas; enquanto Villares e Rocha (1950) registraram, para novilhos observados entre 6 e 19 horas, 8,56 horas de pastejo (65,85\% do tempo de observação) no outono e 11,58 (89,10\%) na primavera. Diferenças climáticas estacionais podem alterar os horários e o tempo de pastejo de bovinos (Machado Filho et al., 1993b). Pastagens melhores diminuem o tempo de pastejo (Villares e Rocha, 1950), que neste estudo coincidem com o verão, época das observações.

O maior tempo gasto em uma determinada categoria de atividade levou à diminuição compensatória em outras categorias. De fato, o aumento no tempo dedicado às atividades sexuais pode ter causado a diminuição do pastejo, bem como de outras atividades, como evidenciado pelo grupo sob maior incidência de cio, no qual os touros pastaram menos nos dias de maior concentração de atividades sexuais, em torno do primeiro e segundo dias de observação. Além disto, os touros sob baixa e alta incidência de cio tiveram picos de pastejo diferenciados: entre 6 e 9, 15 e 18 (maior pico) e 21 e 24 horas para os de baixa incidência de cio e entre 0 e 3, 6 e 9 e 18 e 21 horas (maior pico), para os de alta. Destacase ainda a alteração promovida pela ocorrência de serviço completo no lote, com redução do tempo despendido em pastejo e nas interações agonísticas, sendo 2,35 e 2,13 menores do que quando não ocorreu o serviço completo. Houve ainda alteração nos hábitos de pastejo dos touros, pois apenas quando não ocorreu serviço completo no lote os touros apresentaram padrão similar ao observado na literatura (Villares e Rocha, 1950; Raadsma et al., 1983/1984;
Boyd et al., 1989).

À exceção do período do dia, as demais fontes de variação consideradas neste estudo caracterizaram que o aumento nas atividades sexuais de touros se dá com o comprometimento das atividades de manutenção, principalmente o pastejo. Como não houve evidência de ingestão compensatória durante o período de estudo, a longo prazo tal situação poderia resultar em perdas de peso acima do esperado, o que poderia prejudicar a capacidade reprodutiva dos mesmos. Ressalte-se que no período em que as observações foram realizadas, meio da estação de monta, a manifestação de cio já deveria estar baixa, o que pode ser evidenciado pela oferta no lote de baixa incidência que, na realidade, representava a oferta de cio natural num rebanho. Neste caso, os touros apresentavan boas condições corporais (escore 4), o que provavelmente permitiria que estes mobilizassem energia rapidamente, compensando as exigências de um prazo curto de tempo como foi o das observações neste estudo, sem que viessem a apresentar maiores prejuízos na sua capacidade reprodutiva. No entanto, é importante frisar que uma oferta de cio similar à do lote de alta incidência de cio, numa condição não experimental, poderia perpetuar-se por mais tempo, no início da estação. Por exemplo, num lote de acasalamento múltiplo, com três touros e PTV de 1:100, a incidência de cio esperada de 4-5\% levaria a oferta média de 12 fêmeas em cio/dia, nos primeiros 21 dias de estação, se considerarmos que $80 \%$ das fêmeas estivessem ciclando. Sendo assim, seria necessário que estes animais compensassem o baixo tempo de pastejo acionando mecanismos fisiológicos para disponibilizar energia ou talvez outros recursos comportamentais, que não foram avaliados neste estudo, tais como aumento da velocidade de pastejo ou do tamanho da bocada. Infelizmente a avaliação de peso e escore destes animais foi feita apenas no início e final da estação de monta, não permitindo verificar, com acuidade,

Archivos de zootecnia vol. 59, núm. 227, p. 330. 
alterações nas condições corporais que possam ter ocorrido em decorrência do manejo estabelecido durante as observações comportamentais.

A grande oferta de vacas em cio, ainda que por um período curto de tempo, pode caracterizar uma situação de estresse para o touro, o que pode levar inclusive a perda de peso. Sabe-se que o estresse altera a secreção de GnRH, modulando a liberação do hormônio luteinizante (LH) comprometendo a espermatogênese (Barth, 1993; Brooks et al., 1986). Os reflexos poderiam não ser imediatos considerando a duração do ciclo do epitélio seminífero, vindo a comprometer a qualidade seminal já no final da estação reprodutiva. Santos et al. (2004) observaram, em touros submetidos à PTV de 1:100, perdas na qualidade seminal aspectos físicos do sêmen (motilidade, vigor, turbilhonamento e concentração espermática), mas não registraram variações no perímetro escrotal ou na morfologia espermática. O impacto de situações desta natureza sobre a performance dos reprodutores ainda precisa ser mais bem estudado.

A diminuição das interações agonísticas entre os machos sob alta oferta de cio, provavelmente ocorreu devido a maior oferta de fêmeas receptivas o que diminuiu os efeitos da competição social. Situações não controláveis, como interações com touros vizinhos de cerca, foram, provavelmente, responsáveis pelas interações significativas observadas entre hierarquia o dia de observação e período do dia.

Outras atividades, que englobaram predominantemente o ócio, mas também beber água, lamber sal e deslocamento, foram associadas com a atividade sexual. A incidência de cio apresentou efeito significativo sobre o tempo em outras atividades e a interação significativa entre hierarquia e dia de observação sugere que os touros dominantes e subordinados, diante de uma maior demanda por atividades sexuais nos primeiros dias de observação, compensaram aumentando o descanso nos dias subseqüentes em que não havia fêmea em cio. Estes resultados não permitem sustentar esta inferência, pois o descanso não foi diretamente medido neste estudo.

Touros dominantes gastaram mais tempo com atividade sexual que seus subordinados, independente da incidência de cio no lote. As oportunidades para abordagem sexual no lote sob alta incidência de cio ocorreram muito concentradas no tempo. Isso pode ter ocasionado confundimento, levando à interações significativas da incidência de cio com a ordem do dia e com o período do dia. Por isso, conclusões sobre variação das atividades gerais considerando a ordem social dos touros sob alta incidência de cio ficaram comprometidas.

\section{CONCLUSÕES}

Durante a estação de monta os touros priorizam as atividades sexuais, com riscos de comprometimento de suas atividades de manutenção (alimentação e descanso).

\section{AGRADECIMENTOS}

Ao Conselho Estadual de Ciência e Tecnologia, vinculado ao Governo do Estado Mato Grosso do Sul, pelo apoio financeiro. Ao CNPq e à FUFMS pelas Bolsas de Iniciação Científica. À Fazenda Bodoquena S/A, Agropecuária Nove de Ouro e Fazenda Nhumirim da EMBRAPAPantanal pelo apoio integral ao projeto de pesquisa, através de auxílio na mão de obra, infraestrutura e financeiro neste projeto.

\section{BIBLIOGRAFIA}

Barth, A.D. 1993. Insights to the pathogenesis of sperm abnormalities in bulls. Rev. Bras. Reprod. Anim., 4: 1-14.

Blockey, M.A.B. 1978. The influence of serving capacity of bulls on herd fertility. J. Anim. Sci., 46: 589-595.

Archivos de zootecnia vol. 59, núm. 227, p. 331. 
Blockey, M.A.B. 1979. Observations on group mating of bulls at pasture. Appl. Anim. Ethol., 5: 15-34.

Boyd, G.W., Lunstra, D.D. and Corah, L.R. 1989. Serving capacity of crossbred yearling beef bulls. II. Summer grazing activity and body temperature patterns during average and heavy mating loads at pasture. J. Anim. Sci., 67: 7279.

Brooks, A.N., Lamming, G.E. and Haynes, N.P. 1986. Endogenous opioids peptides and the control of gonadotrophin secretion. Res. Vet. Sci., 41: 285-299.

Chenoweth, P.J. 1981. Libido and mating behavior in bulls, boars and rams: A review. Theriogenology, 16: 155-177.

Costa e Silva, E.V. 1994. Capacidade reprodutiva de touros Nelore: exame andrológico, teste de comportamento sexual e desafio de fertilidade. Dissertação, Mestrado em Medicina Veterinária. Escola de Veterinária da UFMG. Belo Horizonte. $102 \mathrm{pp}$.

Costa e Silva, E.V. 1995. Limites dos touros da raça Nelore. O desafio da fertilidade. Em: Symposium - o Nelore do século XXI, 3. Associação dos Criadores de Nelore do Brasil. Palestras... Ribeirão Preto. Ribeirão Preto, SP. pp. 39-43.

Crudeli, G.A., Fonseca, V.O., Costa e Silva, E.V. e Hermanny, A. 1990. Comportamiento sexual de toros Nelore (Bos taurus indicus): efecto de la capacidad de servicio sobre la tasa de fertilidad del rodeo. Cabia, 21: 20-26.

Fonseca, V.O., Crudeli, G.A., Costa e Silva, E.V. e Hermanny, A. 1991. Potencial reprodutivo de touros da raça Nelore (Bos taurus indicuas) em monta natural: proporção touro:vaca de 1:40 e fertilidade. Rev. Bras. Reprod. Anim., 15: 103108.

Machado Filho, L.C.P., Ribeiro, J.A.R., Werner, D., Borges, A.L., Bridi, A.M., Fronza, J. e Panizi, M 1993a. Estudo do comportamento de três tipos étnicos de bovinos: Crioulo Lageano, Charolês e Nelore em campo nativo no inverno e no verão. Em: Encontro anual de Etologia, 11. Anais... Ribeirão Preto: Logis Summa Ltda. Bauru, SP. $248 \mathrm{pp}$.

Machado Filho, L.C.P., Ribeiro, J.A.R., Werner, D.,
Borges, A.L., Bridi, A.M., Fronza, J. e Panizi, M. 1993b. Estudo da variação do comportamento da raça bovina crioulo Lageano no inverno e no verão. Em: Encontro anual de Etologia, 11. Anais... Ribeirão Preto: Logis Summa Ltda. Bauru, SP. 249 pp.

Mader, D.R. and Price, E.O. 1984. The effects of sexual stimulation on the sexual performance of Hereford bulls. J. Anim. Sci., 59: 294-300.

Pineda, N.R., Fonseca, V.O. e Proença, R.V. 2000. Potencial reprodutivo de touros Nelore: libido, capacidade de serviço e eficiência em acasalamentos com elevada proporção touro:vaca. Rev. Bras. Reprod. Anim., 24: 4451.

Raadsma, H.W., Edey, T.N. and Bindon, B.M. 1983/ 1984. Behaviour and mating performance of paddock-mated beef bulls. Anim. Reprod. Sci., 6: 153-165.

Rosa, A.N. 2007. Recursos genéticos e planos de melhoramento em gado de corte. Em: Curso de melhoramento de gado de corte da EMBRAPAGENEPLUS, 17. Anais...Campo Grande. Embrapa Gado de Corte. Campo Grande-MS.

Sampaio, I.B.M. 1998. Estatística aplicada à experimentação animal. Fundação de Ensino e Pesquisa em Medicina Veterinária. Belo Horizonte. 221 pp.

Santos, M.D., Torres, C.A.A., Ruas, J.R.M., Guimarães, J.D. e Silva Filho, J.M. 2004. Potencial reprodutivo de touros da raça Nelore submetidos à diferentes proporções touro:vaca. Arq. Bras. Med. Vet. Zootecn., 56: 497-503.

SAS. 2005. Statistical Analysis system user's guide: Stat., Version 6.11. Cry: SAS Institute. Cary. NC. USA.

Sereno, J.R.B., Costa-e-Silva, E.V. e Mores, C.M. 2000. Redução da proporção touro:vaca no Pantanal Mato-grossense. EMBRAPA Pantanal. Corumbá. 24 pp. (Circular técnica, 26).

Valle, E.R., Encarnação, R.O., Schenk, J.A.P. e Curvo, J.B.E. 1994. Duração do cio e momento de ovulação em vacas Nelore. Rev. Bras. Zootecn., 23: 852-858.

Villares, J.B. e Rocha, G.L. 1950. Climatologia zootécnica $x$ contribuição para o estudo dos hábitos bovinos nas e pastagens tropicais. Bol. Ind. Animal, 11: 3-22.

Archivos de zootecnia vol. 59, núm. 227, p. 332. 